\title{
Anatomical insights into modified posterior-inferior line in patients with atrial fibrillation: Implications in left atrial posterior wall isolation
}

Xiaofeng $\mathrm{Lu}^{1}$, Shi Peng ${ }^{2}$, Xiaoyu $\mathrm{Wu}^{2}$, Genqing Zhou ${ }^{2}$, Yong Wei ${ }^{3}$, Lidong $\mathrm{Cai}^{2}$, Juan Xu ${ }^{2}$, $\mathrm{Yu} \mathrm{Ding}^{4}$, Songwen $\mathrm{Chen}^{5}$, and Shaowen $\mathrm{Liu}^{4}$

${ }^{1}$ Shanghai General Hospital, Shanghai Jiao Tong University, School of Medicine, China ${ }^{2}$ Shanghai General Hospital, Shanghai Jiao Tong University School of Medicine

3 Shanghai General Hospital, Shanghai Jiao Tong University School of Medicine ${ }^{4}$ Shanghai General Hospital, Shanghai Jiao Tong University, School of Medicine

${ }^{5} 1$. Department of Cardiology, Shanghai General Hospital, Shanghai Jiao Tong University, School of Medicine.

September 10, 2020

\begin{abstract}
Introduction Left atrial posterior wall (LAPW) isolation may be performed as an additional atrial fibrillation (AF) ablation strategy based on pulmonary vein isolation. A modified posterior-inferior line (MPL) was proposed for reducing esophageal injury. The aim of this study was to evaluate the anatomical characteristics of the MPL, compared with the CPL. Methods and Results Multidetector computed tomography was performed in 102 consecutive AF patients (male/female $=60 / 42$ ) preoperative, and the parameters were evaluated as follows: the distance from MPL and CPL to the esophagus, fat pad presence and thickness in the course of MPL and CPL, and the esophageal route below CPL. The average distance from the MPL to the esophagus was longer than from CPL to the esophagus $(3.7 \pm 1.5 \mathrm{~mm}$ vs $1.7 \pm 0.4 \mathrm{~mm}, \mathrm{P}<0.001)$. Fat pad presence was higher in the course of MPL than CPL. The myocardium tissue and fat pad under MPL was thicker than under CPL (2.9 $\pm 1.1 \mathrm{~mm}$ vs 1.6 $\pm 0.3 \mathrm{~mm}, \mathrm{P}<0.001 ; 1.4 \pm 0.6 \mathrm{~mm}$ vs $0.9 \pm 0.2 \mathrm{~mm}, \mathrm{P}<0.001)$, respectively. In patients whose esophagus was unconfined in a triangular space at the left inferior pulmonary vein level, the average distance from MPL to esophagus was longer than the confined patients $(4.0 \pm 1.7 \mathrm{~mm}$ vs $3.2 \pm 1.0 \mathrm{~mm}, \mathrm{P}=0.001)$. Conclusion The MPL was far away from the esophagus with thicker myocardium tissue and more fat pad than the CPL; thus, MPL could serve as a favorable alternative in linear ablation for LAPW isolation.
\end{abstract}

Anatomical insights into modified posterior-inferior line in patients with atrial fibrillation: Implications in left atrial posterior wall isolation

Xiaofeng Lü\#1, MD; Peng Shi\#1, MD; Xiaoyu Wu ${ }^{1}$, MD; Genqing Zhou ${ }^{1}$, MD; Yong Wei ${ }^{1}$, MD, PhD; Lidong Cai ${ }^{1}$, MD, PhD; Juan Xu ${ }^{1}$, MD, PhD; Yu Ding ${ }^{1}$, MD; Songwen Chen*1, MD, PhD; Shaowen Liu*1, MD, PhD.

Department of Cardiology, Shanghai General Hospital, School of Medicine, Shanghai Jiao Tong University, Shanghai, China.

\# Xiaofeng Lu and Peng Shi share the first authorship

*Corresponding authors 
Songwen Chen/Shaowen Liu, Department of Cardiology, Shanghai General Hospital, School of Medicine, Shanghai Jiao Tong University, No. 100, Haining Road, 200080 Shanghai, China. Email: chensongwen@hotmail.com/shaowen.liu@hotmail.com

Abstract

\section{Introduction}

Left atrial posterior wall (LAPW) isolation may be performed as an additional atrial fibrillation (AF) ablation strategy based on pulmonary vein isolation. A modified posterior-inferior line (MPL) was proposed for reducing esophageal injury. The aim of this study was to evaluate the anatomical characteristics of the MPL, compared with the CPL.

\section{Methods and Results}

Multidetector computed tomography was performed in 102 consecutive AF patients (male/female $=60 / 42$ ) preoperative, and the parameters were evaluated as follows: the distance from MPL and CPL to the esophagus, fat pad presence and thickness in the course of MPL and CPL, and the esophageal route below CPL. The average distance from the MPL to the esophagus was longer than from CPL to the esophagus $(3.7 \pm$ $1.5 \mathrm{~mm}$ vs $1.7 \pm 0.4 \mathrm{~mm}, \mathrm{P}<0.001)$. Fat pad presence was higher in the course of MPL than CPL. The myocardium tissue and fat pad under MPL was thicker than under CPL $(2.9 \pm 1.1 \mathrm{~mm}$ vs $1.6 \pm 0.3 \mathrm{~mm}, \mathrm{P}<$ $0.001 ; 1.4 \pm 0.6 \mathrm{~mm}$ vs $0.9 \pm 0.2 \mathrm{~mm}, \mathrm{P}<0.001)$, respectively. In patients whose esophagus was unconfined in a triangular space at the left inferior pulmonary vein level, the average distance from MPL to esophagus was longer than the confined patients $(4.0 \pm 1.7 \mathrm{~mm}$ vs $3.2 \pm 1.0 \mathrm{~mm}, \mathrm{P}=0.001)$.

\section{Conclusion}

The MPL was far away from the esophagus with thicker myocardium tissue and more fat pad than the CPL; thus, MPL could serve as a favorable alternative in linear ablation for LAPW isolation.

Keywords: Catheter ablation - atrial fibrillation, Enhanced multidetector computed tomography (CT), Left atrial posterior wall (LAPW) isolation, Modified posterior-inferior line (MPL), Conventional posterior line $(\mathrm{CPL})$

\section{INTRODUCTION}

Pulmonary vein (PV) isolation has been considered as the cornerstone for catheter ablation in the treatment of atrial fibrillation (AF). ${ }^{1,2}$ However, the ablation of persistent AF (PsAF) remains a challenge for clinical practice. Due to the underlying structural, electrical, and autonomic pathologic remodeling of PsAF, the success rate could decrease over time. Although reports have indicated that atrial and pulmonary vein (PV) muscle sleeve microstructural remodeling is present in AF establishing a vulnerable substrate for $\mathrm{AF}$ maintenance, there are more non-PV foci atrial substrates for the maintenance of $\mathrm{AF} .{ }^{3}$ Non-PV foci commonly arise from the left atrial posterior wall (LAPW), left atrial appendage, and the superior vena cava, and so on. ${ }^{4}$ Based on PV isolation, additional LAPW isolation has been reported as an effective strategy for improving the ablation outcomes for PsAF. ${ }^{5,6}$ Furthermore, LAPW isolation has also been performed for paroxysmal AF (PAF). ${ }^{5} 7$ One of the classic LAPW isolation approaches involves a wide antral PV isolation, followed by roofline and conventional posterior line (CPL). The CPL is usually connected to the most inferior margin of the inferior $\mathrm{PVs}^{6,8}$ or the posterior-inferior part of two inferior PVs. ${ }^{9,}{ }^{10}$ Due to the anterior aspect of the esophagus was directly opposed to the LAPW, the CPL could not avoid touching the esophagus. ${ }^{11}$ Therefore, it is predictable that the adjunct ablation on LAPW, theoretically, increases the risk of esophageal injury and even result in an atrio-esophageal fistula (AEF). Previous studies investigated the myocardium of LAPW and tissue layers between LAPW and esophagus by multidetector computed tomography $(\mathrm{CT})$, intracardiac echocardiography, specimen and histological analysis. ${ }^{12-14}$ However, only a few studies have compared the distance from the endocardium of LAPW to the esophagus between different levels of LAPW. ${ }^{12,13}$ In this study, a modified approach of LAPW isolation was proposed by replacing CPL with a modified posterior-inferior line (MPL), which was in a lower region of LAPW than CPL. 
The anatomical difference between CPL and MPL were compared in patients with AF using enhanced multidetector CT analysis.

\section{METHODS}

\subsection{Study population}

This study enrolled a total of 102 consecutive patients (male/female $=60 / 42$ ) with paroxysmal AF (PAF; $\mathrm{n}=31$ ) or persistent AF (PsAF; $\mathrm{n}=71$ ), who was referred to our clinic at Shanghai General Hospital for radiofrequency catheter ablation from January to June 2018. The PsAF and PAF were defined according to the European Society of Cardiology (ESC) guidelines. ${ }^{3}$ Our patients' exclusion criteria were as follows: had previously undergone left atrial ablation procedures, had previous cardiac or mediastinal surgery, and those who were unable to receive enhanced CT examination.

The study protocol was approved by the Institutional Ethics Review Board. Each patient was provided written informed consent before the procedure.

\subsection{Cardiac CT scan}

All patients received a contrast-enhanced CT scan 1 to 2 days before the ablation procedure. CT examinations of the left atrium (LA), PVs, aorta, and esophagus were performed on a 256-multidetector spiral CT scanner (Revolution, GE Healthcare, Milwaukee, USA). The intravenous contrast (Iopamidol 370, Shanghai Pharmaceuticals Sine, China) was administered with a bolus track technique at a rate of $4 \mathrm{ml} / \mathrm{s}$ through an antecubital vein. The scanning parameters are listed as follows: slice thickness $0.625 \mathrm{~mm}$, collimation of $64 \mathrm{x}$ $0.5 \mathrm{~mm}$, the gantry rotation time was $350 \mathrm{~ms}$, tube voltage $120 \mathrm{kV}$, tube current $200-500 \mathrm{~mA}$ (Smart control). The scanning procedure was started after the CT value of the center of the LA chamber (region of interest) was higher than 150, and all the CT angiographic images were obtained in the craniocaudal direction with a covering of the heart during a single breath-hold (within 3 seconds). All data were transferred to GE Advantage Windows Workstation (AW 4.6 system) for post-image processing. A volume rendering technique was performed to obtain three-dimensions (3D) images of the LA, and all multiplanar reconstruction images were gathered. The spatial resolution in all the $3 \mathrm{D}$ images was $0.30 \mathrm{~mm}$.

\subsection{Determination of the posterior lines and esophageal routes}

The PV ostia was defined by an abrupt angular change between the LA and PV planes. Circumferential ablation lesions were created by encircling the ipsilateral left and right PV ostia. The roofline was made by connecting both sides of the PV isolation ring at the top level. Then the PV isolation ring, roofline, along with both CPL and MPL posterior lines, were shown on the 3D CT images (Figure 1). CPL was defined as a straight line connecting both sides of the inferior PVs at the bottom level, as established in previous studies. ${ }^{6}, 8$ MPL was designed as follows: (1) A curve inferior to CPL on the endocardium of LA from posterior-anterior view and a smile was formed, where MPL corresponded to the curve of the lower lip, (2) the most inferior point of MPL was located at the level of the midpoint, where a line connected the midpoint of CPL and the most inferior point of LA, (3) MPL started from the anterior-inferior part of the left PV isolation ring and ended at the bottom level of the right PV, (4) the curve was bilateral symmetry. CT images in the axial, sagittal, and coronal planes were evaluated to determine the route and outline of the esophagus along the posterior LA wall. ${ }^{12,}{ }^{15}$ The posterior line segment that touched by the esophagus was divided into 2 equal portions by 3 points, respectively. According to the MPL, the esophageal route was classified into three types (Figure 1): type A esophageal route covered the start portion of MPL (only descending limb of the curve), type B esophageal route covered the middle portion of MPL (both descending and ascending limb of the curve), and type C esophageal route covered the end portion of the MPL (only ascending limb of the curve). Esophageal route type 1 was defined as the esophagus closer to the left inferior PV than to the right inferior PV, and esophageal route type 2 was defined as the esophagus closer to the right inferior PV than to the left inferior PV, as described previously. ${ }^{16}$ The topological locations of the esophagus among LAPW, inferior PVs, aorta, and spine, were determined at the left inferior PV level and MPL level. 


\subsection{Measurements}

Measurements of the parameters were made using a digital caliper on each posterior line under predefined points $(\mathrm{CPL}=\mathrm{a}, \mathrm{b}, \mathrm{c}$ and $\mathrm{MPL}=\mathrm{d}, \mathrm{e}, \mathrm{f})$, which are segments that touched the esophagus, with their related esophageal routes are as follows: (1) the shortest distance from the endocardium of LAPW to the anterior border of the esophagus. (2) The thickness of the fat pad, if it existed. (3) The thickness of LAPW myocardium. (4) The distance between the esophagus and ipsilateral inferior PV ostia and the shortest distance from LA to the spine at the left inferior PV level. The distance from the endocardium of LAPW to the esophagus was measured from the density of the intra-cavitary contrast that filled the LA to the anterior border of the esophagus (Figure 2). The presence of a fat pad between the LAPW and the esophagus was identified by an abrupt change in the signal density. The thickness of the LAPW myocardium was measured from the density of the contrast agent in the LA to the fat pad or the outline of the anterior esophageal wall if no fat pad was present. All parameters were determined independently by two cardiologists using axial planes and 3D CT images. If there were discrepancies between those results, they were adjudicated by a third investigator.

\subsection{Definition of measurements}

The average and minimum distance from each posterior line to the esophagus and the average and minimum thickness of LAPW myocardium under each posterior line were defined as the mean and minimum value of 3 predefined points (a, b, c and d, e, f) on CPL and MPL, respectively. The average thickness of the fat pad was defined as the mean value of the predefined point that appeared with the fat pad on each course of the posterior line, respectively. In some cases, the esophagus was enclosed by left inferior PV, descending aorta, and vertebral body in a triangular space at left inferior PV level (defined as the confined group), while in other cases the esophagus was not enclosed by LAPW, descending aorta, and the vertebral body simultaneously in a triangular space at left inferior PV level (defined as the unconfined group) (Figure 3 and Figure 2A).

\subsection{Statistical analysis}

Continuous variables were expressed as mean +- standard deviation. Discrete variables were presented as percentages. Univariate comparisons among the variables were made using the chi-squared $\left(\chi^{2}\right)$ test for categorical variables and the Student's t test for continuous variables. A one-way ANOVA followed by Bonferroni's post hoc test was performed to evaluate the statistical differences of variables among the groups for continuous variables. Pearson correlation analysis was performed for multiple continuous variables. All tests were two-tailed. $\mathrm{P}<0.05$ was considered statistically significant. All statistical analyses were performed by SPSS (SPSS Inc., version 21.0, Chicago, IL, USA).

\section{RESULTS}

\subsection{Patient Characteristics}

The mean age of the patients was $63.1 \pm 10.6$ years. Structural heart disease was present in one patient with PAF who had hypertrophic cardiomyopathy. No other structural heart disease was found in both AF groups. The mean LA diameter measured by the echocardiogram was $43.3 \pm 5.6 \mathrm{~mm}$. The demographics and clinical characteristics of the patient are shown in Table 1.

\subsection{Anatomical characteristics of CPL and MPL}

The average distance from MPL to the esophagus was farther than from CPL to the esophagus $(4.0 \pm$ $1.7 \mathrm{~mm}$ vs $3.2 \pm 1.0 \mathrm{~mm}, \mathrm{P}<0.001$, Table 1$)$. The fat pad under MPL appeared with higher frequency than that under CPL $(74.5 \%$ vs $18.6 \%, \mathrm{P}<0.001)$ and the average thickness of the fat pad under MPL was thicker than that under CPL $(1.4 \pm 0.6 \mathrm{~mm}$ vs $0.9 \pm 0.2 \mathrm{~mm}, \mathrm{P}<0.001)$. The average thickness of LAPW myocardium under MPL was also thicker than that under CPL $(2.9 \pm 1.1 \mathrm{~mm}$ vs $1.6 \pm 0.3 \mathrm{~mm}, \mathrm{P}<0.001)$.

\subsection{The esophageal route}


For each type of esophageal route below CPL, MPL was observed with a longer average distance to the esophagus $(\mathrm{P}<0.001)$, greater average thickness of the myocardium $(\mathrm{P}<0.001)$, and higher presence of fat pad than that observed in CPL, respectively $(\mathrm{p}<0.001$, Table 2$)$. The minimum distance from the MPL to the esophagus was also longer than that from $\mathrm{CPL}$ to the esophagus $(\mathrm{P}=0.001$, Table 2$)$. However, in the type $\mathrm{C}$ esophageal route, the minimum thickness of myocardium under $\mathrm{CPL}$ was similar to that under MPL $(\mathrm{P}=0.06)$. In each posterior line group, there was no difference in the distance from LAPW to the esophagus relative to the thickness of LAPW myocardium or the presence of a fat pad among the 3 types of the esophageal routes $(\mathrm{P}>0.05)$. Above the level of $\mathrm{CPL}$, the esophageal route type 1 included 93 patients (91.2\%), and esophageal type 2 included 9 patients (8.8\%). Below the level of CPL, all of the type A route (36 patients), all type $\mathrm{B}$ route (53 patients) and only $30.8 \%$ of type $\mathrm{C}$ route $(\mathrm{n}=4 / 13$ patients) to the esophagus had a shorter distance to the left inferior PV than to the right inferior PV. Of the type C esophageal route patients, $69.2 \%(\mathrm{n}=9 / 13)$ had a shorter distance to the right inferior PV than to the left inferior PV.

\subsection{Anatomical difference between confined group and unconfined group}

Precisely, 38.2\% ( $\mathrm{n}=39)$ of the AF patients were designated as confined group, and $61.8 \%(\mathrm{n}=63)$ as unconfined group (Table 3). There were no differences in the AF types, LA diameter, and the average distance from CPL to the esophagus in the confined group, compared with the unconfined group ( $\mathrm{p}>$ 0.05). The shortest distance from the spine to LAPW at the left inferior PV level was shorter in a confined group than the unconfined group $(\mathrm{P}<0.001)$. The average distance from the MPL to the esophagus in the unconfined group was longer than the confined group $(4.0 \pm 1.7 \mathrm{~mm}$ vs $3.2 \pm 1.0 \mathrm{~mm}, \mathrm{P}=0.001$, Table 3$)$. The thickness of the myocardium in the course of MPL in the unconfined group was thicker than the confined group $(3.1 \pm 1.2 \mathrm{~mm}$ vs $2.6 \pm 0.7 \mathrm{~mm}, \mathrm{p}=0.023)$. When $\mathrm{CPL}$ was replaced by MPL, the increased ratio of the distance from the endocardium of the posterior wall to the esophagus was higher in the unconfined group than the confined group $(2.4 \pm 0.9$ times vs $1.9 \pm 0.6$ times, $\mathrm{p}<0.001)$. The presence and the average thickness of the fat pad were similar in both groups $(\mathrm{p}>0.05)$. The average distance from the MPL to the esophagus was longer than from the CPL to the esophagus in both confined and unconfined groups $(3.2 \pm 1.0 \mathrm{~mm}$ vs $1.6 \pm 0.4 \mathrm{~mm}, \mathrm{p}<0.001 ; 4.0 \pm 1.7 \mathrm{~mm}$ vs $1.6 \pm 0.3 \mathrm{~mm}, \mathrm{P}<0.001)$, respectively. Type A esophageal route appeared more frequently in the confined group than the unconfined group $(57.1 \%$ vs $23.9 \%, \mathrm{p}<0.05)$, while Type $\mathrm{C}$ esophageal route appeared more frequently in the unconfined group than the confined group $(19.4 \%$ vs $0 \%, \mathrm{p}<0.05)$.

\subsection{Comparison between PAF and PsAF}

The PAF and PsAF patients were comparable, with no statistical difference in age and gender between them ( $\mathrm{p}>0.05$, Table 1). The LA diameter and body mass index (BMI) was higher in the PsAF than PAF ( $\mathrm{p}$ $<0.05$ ). There was no difference in the esophageal route (above or below CPL) distribution between PAF and PsAF ( $\mathrm{p}>0.05)$. There was no difference in the average distance from CPL or MPL to the esophagus, in the presence of a fat pad in the course of the CPL or MPL or myocardium and fat pad thickness in the course of the CPL or MPL between PAF and PsAF patients ( $p>0.05)$.

\section{DISCUSSION}

\subsection{Major findings}

This present study has relevant clinical implications, summarized as the main findings, as follows: 1) There was a long distance from the MPL to the esophagus, compared with the distance from the CPL to the esophagus. 2) The myocardium and fat pad in the course of MPL was thicker than in the course of CPL; 3) A higher presence of fat pad was noted between the MPL and the esophagus than between CPL and esophagus 4) The MPL was observed to have a longer distance to the esophagus, thicker myocardium, and higher presence of fat pad in each type of esophageal route below the CPL than the CPL. 5) The average distance from MPL to the esophagus in patients with unconfined esophagus in the triangular space at the left inferior PV level was longer than those with confined esophagus in the triangular space at left inferior PV level. Nonetheless, the average distance from the MPL to the esophagus, compared with the CPL was still longer to the esophagus in the confined group. 


\subsection{The anatomical differences between MPL and CPL}

The distance from the posterior line to the anterior aspect of the esophagus was mainly contributed by the myocardium of the LAPW, parietal pericardium, and the fibrofatty tissue. ${ }^{13}$ However, the parietal pericardium was identified via histological examination, whereas it was almost impossible to identify this parameter from the CT images in the narrow space between LAPW and the esophagus. In the CT image analyses, the myocardium and the fat pad were the main evaluating factors for the distances. Previous studies indicated that the CPL overlap with the LAPW might be responsible for the esophageal injury and even the atrial-esophageal fistula (AEF). ${ }^{8}, 17$ Moreover, the location of the AEF was commonly related to the ablation lines of the left inferior PV-LA junctions. ${ }^{17,} 18$ In our study, instead of CPL, we employed MPL, defined by starting at the anterior-inferior part of left PV, which was at a longer distance from the esophagus (Figure 1). Therefore, the esophagus was separated from LAPW by the left inferior PV, which protected it from ablation injury.

\subsection{The LAPW myocardium and the fat pad}

No relationship was found between the LA diameter, age, gender, BMI, type of AF (PAF or PsAF), and the thickness of the LAPW myocardium under CPL and MPL. The thickness of LAPW myocardium could vary from different measurements. A cadaver study reported the myocardium was thicker in the inferior region (close to coronary sinus) than in the middle region (close to inferior PV), and the roofline region was the thinnest. ${ }^{13}$ This result was comparable with our study in which we also observed that the myocardium inferior $\mathrm{PV}$ region corresponded to the lower region of $\mathrm{MPL}$, while the middle region corresponded to the region of CPL. From the CT study, however, Lemola et al ${ }^{12}$ reported that the thickness of LAPW myocardium was $2.2 \pm 0.9 \mathrm{~mm}$ with no difference from superior to inferior regions. Suenari et al ${ }^{19}$ reported that in cases where there were close contacts between the esophagus and left PV atrium, the distance was about 1.79 $\pm 0.22 \mathrm{~mm}$. These measurements indicated that the LAPW musculature was quite thin in the inferior PV region. Indeed, the thickness of LAPW myocardium in the region of CPL almost represents the distance between the endocardium of LAPW and the esophagus, ${ }^{11}$ because a lower fat pad is presented between the $\mathrm{CPL}$ and the esophagus. It was reported the fat pad was mainly located in the superior and inferior part of LAPW ${ }^{19}$ and it was thinnest at the level between the orifices of the inferior $\mathrm{PV}^{13}$ where the CPL was located. Consistent with our study, only $18.6 \%$ of CPL with a fat pad was observed, whereas $74.5 \%$ of MPL with a fat pad was noticeable. Therefore, the higher presence of a fat pad in the MPL might serve as a protection of esophageal ablation injury.

\subsection{Implications for LAPW isolation}

Reducing the ablation power and duration of radiofrequency application was commonly performed during LAPW ablation in an attempt to avoid excessive damage to the esophagus. However, taking these measures was empirical, and the ability of the procedure to ensure safety was hard to demonstrate due to the very low incidence of AEF. Thus, we envisaged that it might be safer to perform MPL instead of CPL since MPL was relatively farther from the esophagus than CPL; moreover, we considered the higher presence of the fat pad might protect the esophagus from ablation injury. ${ }^{12}$ The electrical isolation of the LAPW by linear lesions is always technically difficult because of the complex structure of the LA musculature and the unavoidable gaps in the ablation line. ${ }^{20}$ For the goal of electrically isolating LAPW, more energy is expected to be delivered on the ablation line. Lee et al. ${ }^{21}$ reported that the achievement of the bidirectional block of the CPL in LAPW isolation was not mandatory due to the risk of esophageal injury and that additional LAPW focal ablation was needed. McLellan et al $^{6}$ reported a similar adjunct radiofrequency ablation at the midportion of CPL by using adenosine challenge. It was predictable that the risk of esophageal injury might be increased by the adjunct ablation on CPL. Further, the MPL ran much inferior, compared with CPL, where the inferior left ganglionated plexi (GP) and inferior right GP were anatomically located, ${ }^{22}$ hence, it is possible that a modification effect of GP might reduce AF recurrence when MPL is performed. One of the predisposing factors of AEF has been suggested, which is a thin posterior LA wall with reduced soft tissue between the esophagus and the LA in slim patients. ${ }^{23}$ In view of the thicker LAPW myocardium and fat pad in the course of MPL, we found it relatively safer to perform MPL instead of CPL. 
4.5 The impact of the esophageal route and periesophageal structures on the distance between the endocardium of LA and the esophagus

Tsao et $\mathrm{al}^{16}$ and Maeda et $\mathrm{al}^{15}$ classified the esophageal route into two major types according to the inferior PVs. Most of the esophageal route ( $88 \%$ to $90 \%$ ) was categorized into type 1 , which was similar in our study $(91.2 \%)$. MPL ran much inferior to CPL when it touched the LAPW. In our study, a thicker myocardium and higher presence of fat pad were identified in the course of MPL than in all the 3 types of esophageal routes below CPL. We also focused on the esophagus compressed in a triangle space at the left inferior PV level because the esophagus vulnerability to injury when LAPW ablation is performed ${ }^{11}$ possibly, in part, attributable to the short distance from the LAPW to the esophagus and the confinement by periesophageal structures. Reports have shown that higher peak esophageal luminal temperatures were noted with ablation in AF patients whose esophagus were confined by LA, aorta, and the vertebral body. Thus, esophageal confinement might also be a risk factor for the development of AEF. ${ }^{24}$ Our results show that $38 \%$ of the AF patients were among the confined group, which indicated that this kind of topological esophagus location is not rare. However, none of the esophagus was enclosed by LA, aorta, and vertebral body at the MPL level. Thereby, the longer the distance of the MPL from the esophagus, the better it is to avoid ablation at such a vulnerable area, and the subsequent chance to reduce the risk of esophageal injury. However, we still need to pay more attention to the shorter distance from MPL to the esophagus displayed by the confined group, compared with the unconfined group, as it was relatively more accessible and safer when we perform MPL in the unconfined group than in the confined group.

\subsection{Limitations}

Despite the success in the outcomes of our study, it does not preclude limitations: First, it is well known that the esophagus is a movable organ, and its exact location might vary at the time of ablation. In this study, the CT images were obtained before the AF ablation procedure, which might not give the actual location of the esophagus intraoperatively. However, another study ${ }^{25}$ showed high concordance between the esophageal location on CT scan and the Carto map, especially in the middle and inferior portion of the LA. Second, the standard ablation parameter of MPL was not uniform. Therefore, a more appropriate ablation parameter such as power, time, and inter-point distance needs to be investigated. Third, more clinical studies are required to confirm the efficacy and safety of the MPL ablation in LAPW isolation. Currently, a multicenter randomized controlled trial (ChiCTR1800015747, Chinese Clinical Trial Registry) is in the recruiting status.

\section{CONCLUSION}

The anatomical superiority of longer distances, thicker LAPW myocardium, and the presence of higher fat pad between the MPL and the esophagus might protect it from AF ablation injury. Therefore, the MPL could serve as a favorable alternative for linear ablation in LAPW isolation.

\section{ACKNOWLEDGMENTS}

This study was supported by the National Natural Science Foundation of China (No. 81970273), the Clinical Research Plan of Shenkang Hospital Development Center of Shanghai (No. 16CR1015A), and the Clinical Research Plan of Shanghai Municipal Health Commission (No. 202040461).

\section{DISCLOSURE}

None.

\section{CONFLICT OF INTERESTS}

The authors declare no conflict of interest 

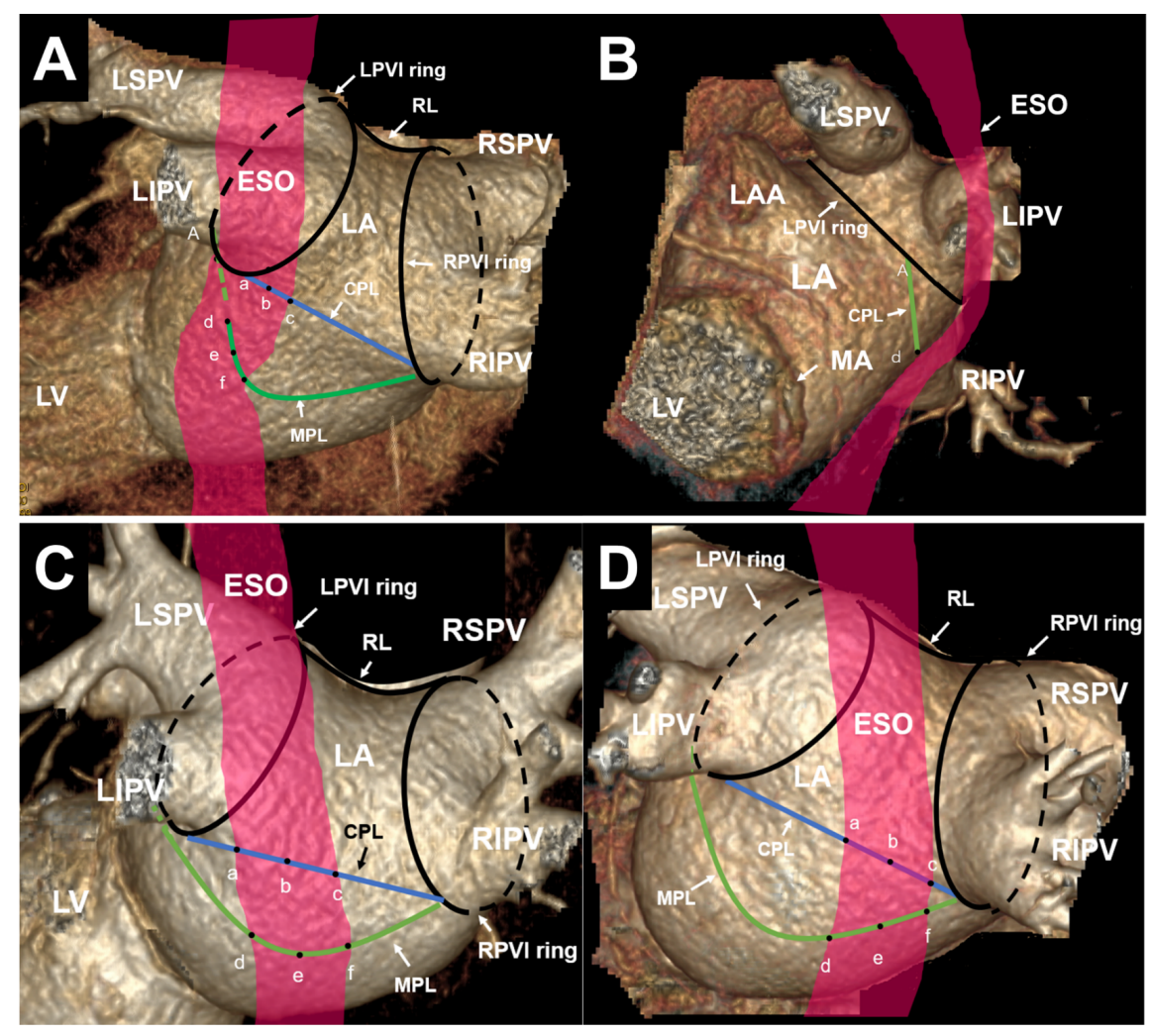

FIGURE 1 The relationship between the esophageal route and the CPL, as well as the MPL from threedimensional (3D) volume rendering computed tomography (CT) images. The CPL (blue) and the MPL (green) were both shown on LAPW. The CPL or the MPL segment that touched the esophagus was divided into two equal parts by 3 points, respectively ( $a, b, c$ and d, e, f). A: Type A esophageal route covered the start portion of MPL (only descending branch of this line) B: The left lateral view of the same patient of panel A showed the start portion of MPL was separated from the esophagus by LIPV. C: Type B esophageal route covered mid part of MPL (both descending and ascending branches of this line). D: Type C esophageal route covered the end portion of MPL (only ascending branch of this line). We measured the shortest distance from every point to the anterior border of the esophagus. The esophagus in panel A and panel $\mathrm{C}$ were also categorized into esophageal route type 1, while the esophagus in panel D was categorized into esophageal route type 2. DA, descending aorta; ESO, esophagus; LA, left atrium; LAA, left atrial appendage; LIPV, left inferior pulmonary vein; LL, left lateral; LPVI, left pulmonary vein isolation; LSPV, left superior pulmonary vein; LV, left ventricle; MA, mitral annulus; MPL, modified posterior-inferior line; CPL, conventional posterior line; RIPV, right inferior pulmonary vein; RPVI, right pulmonary vein isolation; RSPV, right superior pulmonary vein. 


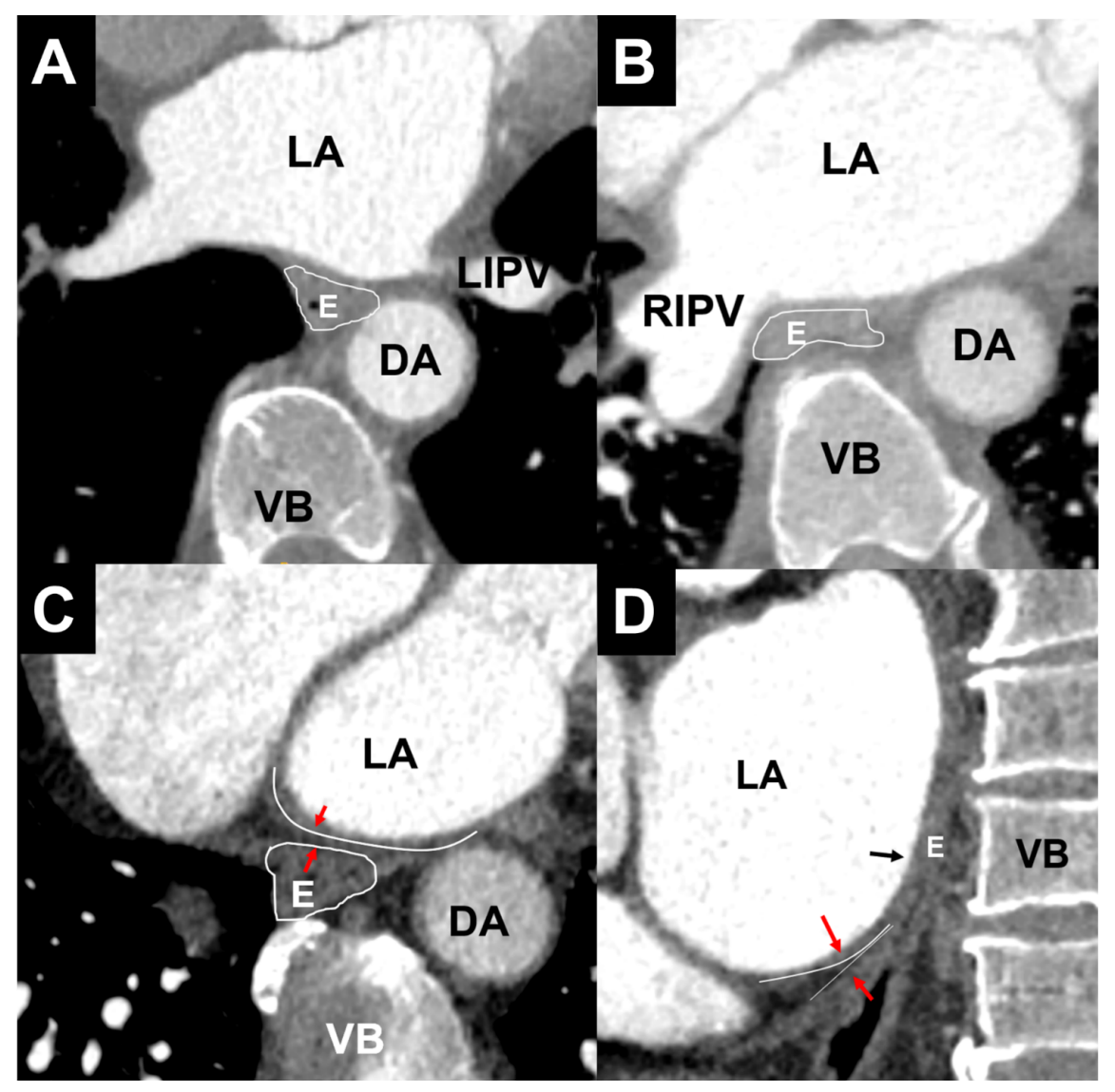

FIGURE 2 Computed tomography (CT) images of LA, esophagus, and periesophageal structures. Panel $\mathrm{A}$ and panel B were axial CT images at the CPL level. Panel A, the esophagus was close to LIPV; Panel B, the esophagus was close to RIPV. No fat pad between LAPW and esophagus was identified on both panel A and panel B. The esophagus was not enclosed by the vertebral body, descending aorta and LAPW on both panel A and panel B; Panel C, a fat pad (red arrow) between LAPW and esophagus was identified at the MPL level. Panel D, sagittal CT image of LA and esophagus, a fat pad (red arrow) was identified at an inferior level (MPL level), while no fat pad was identified at CPL level (black arrow). DA, descending aorta, E, esophagus, LA, left atrium; CPL, conventional posterior line; MPL, modified posterior-inferior line; LIPV, left inferior pulmonary vein; RIPV, right inferior pulmonary vein; VB, vertebral body; LAPW, left atrial posterior wall. 


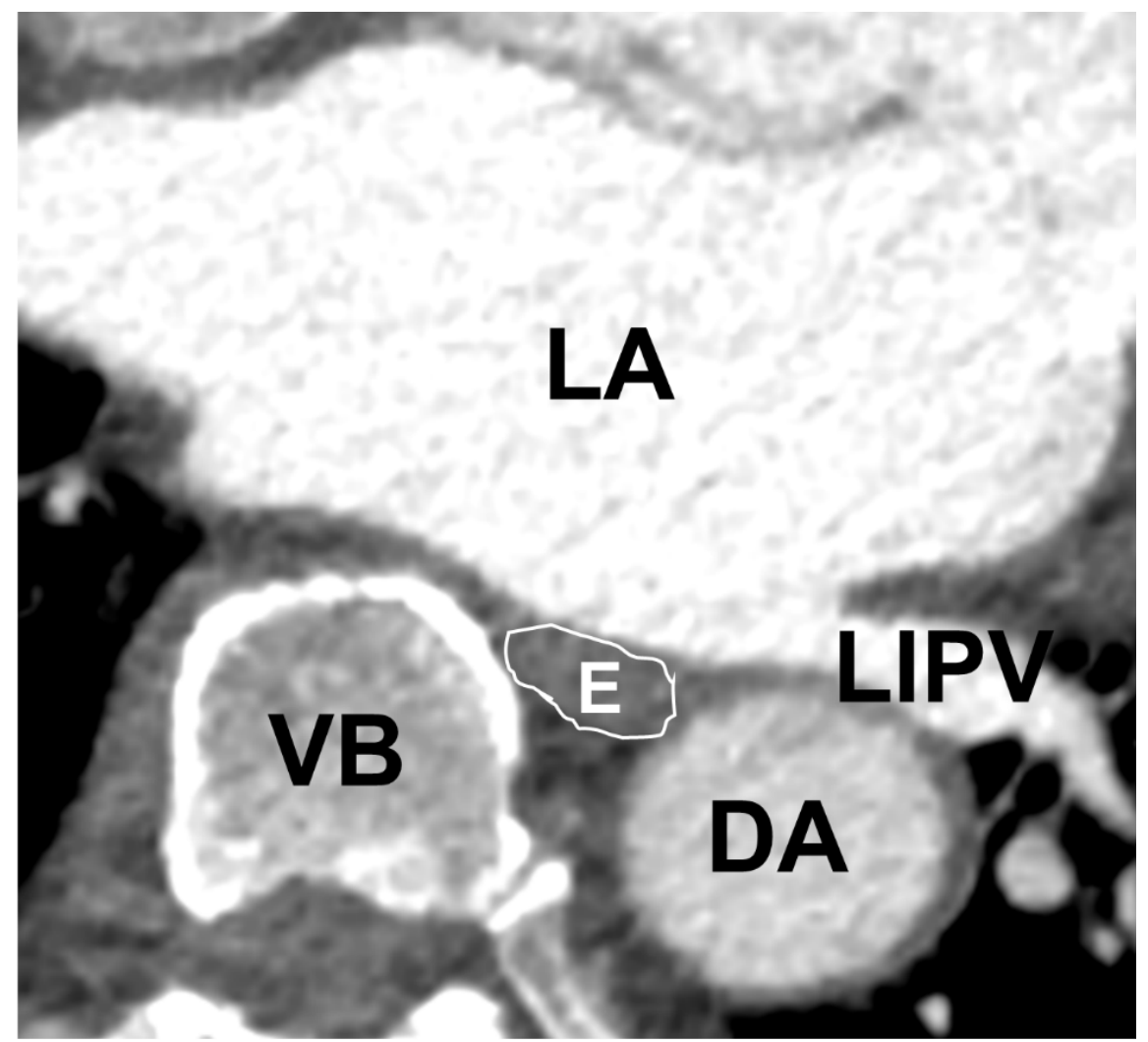

FIGURE 3 The esophagus was confined by LIPV, vertebral body, and descending aorta in a triangular space at the LIPV level. In this group, the distance from MPL to the esophagus was shorter than that in the esophagus, unconfined in a triangular space at the LIPV level (Figure 2A). DA, descending aorta; E, esophagus; LA, left atrium; LIPV, left inferior pulmonary vein; VB, vertebral body; MPL, modified posterior-inferior line.

Table 1.

\begin{tabular}{|c|c|c|c|c|}
\hline & Total $(102)$ & $\operatorname{PAF}(31)$ & PsAF (71) & $\mathrm{P}$ Value \\
\hline Male Gender, n (\%) & $60(58.8 \%)$ & $16(51.6 \%)$ & $44(62.0 \%)$ & 0.384 \\
\hline Age, years & $63.1 \pm 10.6$ & $61.0 \pm 9.5$ & $64.0 \pm 11.0$ & 0.189 \\
\hline $\mathrm{BMI}, \mathrm{kg} / \mathrm{m} 2$ & $25.9 \pm 3.4$ & $24.7 \pm 3.4$ & $26.5 \pm 3.2$ & 0.014 \\
\hline LA diameter, $\mathrm{mm}$ & $43.3 \pm 5.6$ & $39.1 \pm 5.7$ & $45.1 \pm 4.5$ & $<0.001$ \\
\hline Coronary artery disease, $\mathrm{n}(\%)$ & $7(6.9 \%)$ & $1(3.2 \%)$ & $6(8.5 \%)$ & 0.337 \\
\hline Hypertension, n (\%) & $50(49.0 \%)$ & $15(48.4 \%)$ & $35(49.3 \%)$ & 0.933 \\
\hline Diabetes, n $(\%)$ & $10(9.8 \%)$ & $4(12.9 \%)$ & $6(8.5 \%)$ & 0.487 \\
\hline Average Distance from CPL to ESO, mm & $1.7 \pm 0.4$ & $1.6 \pm 0.4$ & $1.7 \pm 0.4$ & 0.809 \\
\hline Average Distance from MPL to ESO, mm & $3.7 \pm 1.5$ & $3.6 \pm 1.4$ & $3.8 \pm 1.5$ & 0.525 \\
\hline Fat pad under CPL, n (\%) & $19(18.6 \%)$ & $4(12.9 \%)$ & $15(21.1 \%)$ & 0.414 \\
\hline Thickness of fat pad under CPL, mm & $0.9 \pm 0.2$ & $1.0 \pm 0.3$ & $0.9 \pm 0.2$ & 0.411 \\
\hline Fat pad under MPL, n (\%) & $76(74.5 \%)$ & $22(71.0 \%)$ & $54(76.1 \%)$ & 0.588 \\
\hline Thickness of fat pad under MPL, mm & $1.4 \pm 0.6$ & $1.4 \pm 0.6$ & $1.4 \pm 0.6$ & 0.984 \\
\hline Average thickness of LAPW myocardium under CPL, mm & $1.6 \pm 0.3$ & $1.5 \pm 0.3$ & $1.6 \pm 0.4$ & 0.231 \\
\hline $\begin{array}{l}\text { Average thickness of LAPW myocardium under MPL, mm } \\
\text { Type of Esophageal route above CPL, } \mathrm{n}(\%)\end{array}$ & $2.9 \pm 1.1$ & $2.9 \pm 0.9$ & $2.9 \pm 1.1$ & 0.760 \\
\hline
\end{tabular}




\begin{tabular}{lllll}
\hline & Total (102) & PAF (31) & PsAF (71) & P Value \\
\hline Type 1 & $93(91.2 \%)$ & $28(90.3 \%)$ & $65(91.5 \%)$ & 0.553 \\
Type 2 & $9(8.8 \%)$ & $3(9.7 \%)$ & $6(8.5 \%)$ & \\
Type of Esophageal route below CPL, n (\%) & & & & \\
type A & $36(35.3 \%)$ & $10(32.3 \%)$ & $26(36.6 \%)$ & 0.954 \\
type B & $53(52.0 \%)$ & $17(54.8 \%)$ & $36(50.7 \%)$ & \\
type C & $13(12.7 \%)$ & $4(12.9 \%)$ & $9(12.7 \%)$ & \\
\hline
\end{tabular}

CPL, conventional posterior line; ESO, esophagus; LA, left atrium; LAPW, left atrial posterior wall; LIPV, left inferior pulmonary vein; MPL, modified posterior-inferior line; PAF, paroxysmal atrial fibrillation; PsAF, persistent atrial fibrillation.

Table 2.

\begin{tabular}{llllllll}
\hline & Type A & Type A & P Value & Type B & Type B & P Value & P \\
\hline & CPL & MPL & & CPL & MPL & & \\
Average distance from LAPW to ESO, mm & $1.7 \pm 0.4$ & $3.8 \pm 1.3$ & $<0.001$ & $1.7 \pm 0.4$ & $3.8 \pm 1.7$ & $<0.001$ & 1 \\
Average Thickness of LAPW myocardium, & $1.6 \pm 0.3$ & $2.9 \pm 0.8$ & $<0.001$ & $1.6 \pm 0.3$ & $3.0 \pm 1.3$ & $<0.001$ & 1 \\
Minimum distance from LAPW to ESO & $1.4 \pm 0.4$ & $3.0 \pm 1.1$ & $<0.001$ & $1.4 \pm 0.3$ & $3.1 \pm 1.4$ & $<0.001$ & 1 \\
Minimum thickness of LAPW myocardium & $1.3 \pm 0.3$ & $2.2 \pm 0.7$ & $<0.001$ & $1.3 \pm 0.3$ & $2.4 \pm 1.1$ & $<0.001$ & 1 \\
Presence of fat pad, n (\%) & $7(19.4 \%)$ & $28(77.7 \%)$ & $<0.001$ & $10(18.9 \%)$ & $40(75.4 \%)$ & $<0.001$ & 2 \\
\hline
\end{tabular}

CPL, conventional posterior line; ESO, esophagus; LA, left atrium; LAPW, left atrial posterior wall; MPL, modified posterior-inferior line.

Table 3.

\begin{tabular}{llll}
\hline & $\begin{array}{l}\text { ESO confined group } \\
(\mathrm{n}=39)\end{array}$ & $\begin{array}{l}\text { ESO unconfined group } \\
(\mathrm{n}=63)\end{array}$ & P Value \\
\hline PAF, n (\%) & $\begin{array}{l}11(28.2 \%) \\
28(71.8 \%)\end{array}$ & $\begin{array}{l}20(31.7 \%) \\
43(68.2 \%)\end{array}$ & 0.706 \\
PsAF, n (\%) & $43.1 \pm 5.6$ & 0.580 \\
$\begin{array}{l}\text { LA diameter, mm } \\
\text { Average distance from }\end{array}$ & $1.7 \pm 0.4$ & 0.556 \\
$\begin{array}{l}\text { CPL to ESO, mm } \\
\text { Average distance from }\end{array}$ & $3.2 \pm 1.0$ & $4.0 \pm 1.7$ & 0.001 \\
$\begin{array}{l}\text { MPL to ESO, mm } \\
\text { Average thickness of }\end{array}$ & $1.6 \pm 0.4$ & $1.6 \pm 0.3$ & 0.820 \\
$\begin{array}{l}\text { LAPW myocardium } \\
\text { under CPL, mm }\end{array}$ & & $3.1 \pm 1.2$ & \\
$\begin{array}{l}\text { Average thickness of } \\
\text { LAPW myocardium } \\
\text { under MPL, mm }\end{array}$ & $2.6 \pm 0.7$ & $11(17.5 \%)$ & 0.023 \\
$\begin{array}{l}\text { Fat pad under CPL, } \mathrm{n} \\
\text { (\%) }\end{array}$ & $8(20.5 \%)$ & $0.9 \pm 0.3$ & 0.700 \\
$\begin{array}{l}\text { Thickness of fat pad } \\
\text { under CPL, mm }\end{array}$ & $1.0 \pm 0.2$ & $50(79.4 \%)$ & 0.655 \\
$\begin{array}{l}\text { Fat pad under MPL, } \mathrm{n} \\
\text { (\%) }\end{array}$ & $26(66.7 \%)$ & $1.5 \pm 0.6$ & 0.153 \\
$\begin{array}{l}\text { Thickness of fat pad } \\
\text { under MPL, mm }\end{array}$ & $1.3 \pm 0.6$ & & 0.251 \\
\end{tabular}




\begin{tabular}{llll}
\hline & $\begin{array}{l}\text { ESO confined group } \\
(\mathrm{n}=39)\end{array}$ & $\begin{array}{l}\text { ESO unconfined group } \\
(\mathrm{n}=63)\end{array}$ & P Value \\
\hline $\begin{array}{l}\text { Distance from spine to } \\
\text { LAPW, mm }\end{array}$ & $4.9 \pm 1.3$ & $12.1 \pm 5.7$ & $<0.001$ \\
$\begin{array}{l}\text { Type of Esophageal } \\
\text { route below CPL, } \mathrm{n}\end{array}$ & & \\
$\begin{array}{l}\text { (\%) } \\
\text { Type A }\end{array}$ & $17(43.6 \%)$ & $19(30.2 \%)$ & \\
Type B & $(56.4 \%)$ & $31(49.2 \%)$ & $<0.05$ \\
Type C & $0(0 \%)$ & $13(20.6 \%)$ & $>0.05$ \\
\hline
\end{tabular}

CPL, conventional posterior line; ESO, esophagus; LA, left atrium; LAPW, left atrial posterior wall; LIPV, left inferior pulmonary vein; MPL, modified posterior-inferior line; PAF paroxysmal atrial fibrillation; PsAF, persistent atrial fibrillation.

\section{References:}

1. Calkins H, Hindricks G, Cappato R, Kim YH, Saad EB, Aguinaga L, et al. 2017 HRS/EHRA/ECAS/APHRS/SOLAECE expert consensus statement on catheter and surgical ablation of atrial fibrillation: executive summary. J Interv Card Electrophysiol. 2017;50(1):1-55.

2. Haïssaguerre M, Jaïs P, Shah DC, Takahashi A, Hocini M, Quiniou G, et al. Spontaneous initiation of atrial fibrillation by ectopic beats originating in the pulmonary veins. N Engl J Med. 1998;339(10):659-66.

3. Kirchhof P, Benussi S, Kotecha D, Ahlsson A, Atar D, Casadei B, et al. 2016 ESC Guidelines for the management of atrial fibrillation developed in collaboration with EACTS. Europace : European pacing, arrhythmias, and cardiac electrophysiology : journal of the working groups on cardiac pacing, arrhythmias, and cardiac cellular electrophysiology of the European Society of Cardiology. 2016;18(11):1609-78.

4. Zhao Y, Di Biase L, Trivedi C, Mohanty S, Bai R, Mohanty P, et al. Importance of non-pulmonary vein triggers ablation to achieve long-term freedom from paroxysmal atrial fibrillation in patients with low ejection fraction. Heart Rhythm. 2016;13(1):141-9.

5. Kumagai K, Nakashima H. Noncontact mapping-guided catheter ablation of atrial fibrillation. Circ J. 2009;73(2):233-41.

6. McLellan AJA, Prabhu S, Voskoboinik A, Wong MCG, Walters TE, Pathik B, et al. Isolation of the posterior left atrium for patients with persistent atrial fibrillation: routine adenosine challenge for dormant posterior left atrial conduction improves long-term outcome. Europace. 2017;19(12):1958-66.

7. Chen J, Off MK, Solheim E, Schuster P, Hoff PI, Ohm OJ. Treatment of atrial fibrillation by silencing electrical activity in the posterior inter-pulmonary-vein atrium. Europace. 2008;10(3):265-72.

8. Kim TH, Park J, Uhm JS, Kim JY, Joung B, Lee MH, et al. Challenging Achievement of Bidirectional Block After Linear Ablation Affects the Rhythm Outcome in Patients With Persistent Atrial Fibrillation. J Am Heart Assoc. 2016;5(10).

9. Kumar P, Bamimore AM, Schwartz JD, Chung EH, Gehi AK, Kiser AC, et al. Challenges and Outcomes of Posterior Wall Isolation for Ablation of Atrial Fibrillation. J Am Heart Assoc. 2016;5(9).

10. Sanders P, Hocini M, Jaïs P, Sacher F, Hsu LF, Takahashi Y, et al. Complete isolation of the pulmonary veins and posterior left atrium in chronic atrial fibrillation. Long-term clinical outcome. Eur Heart J. 2007;28(15):1862-71.

11. Cury RC, Abbara S, Schmidt S, Malchano ZJ, Neuzil P, Weichet J, et al. Relationship of the esophagus and aorta to the left atrium and pulmonary veins: implications for catheter ablation of atrial fibrillation. 
Heart Rhythm. 2005;2(12):1317-23.

12. Lemola K, Sneider M, Desjardins B, Case I, Han J, Good E, et al. Computed tomographic analysis of the anatomy of the left atrium and the esophagus: implications for left atrial catheter ablation. Circulation. 2004;110(24):3655-60.

13. Sanchez-Quintana D, Cabrera JA, Climent V, Farre J, Mendonca MC, Ho SY. Anatomic relations between the esophagus and left atrium and relevance for ablation of atrial fibrillation. Circulation. 2005;112(10):14005 .

14. Ren JF, Lin D, Marchlinski FE, Callans DJ, Patel V. Esophageal imaging and strategies for avoiding injury during left atrial ablation for atrial fibrillation. Heart Rhythm. 2006;3(10):1156-61.

15. Maeda S, Iesaka Y, Uno K, Otomo K, Nagata Y, Suzuki K, et al. Complex anatomy surrounding the left atrial posterior wall: analysis with 3D computed tomography. Heart Vessels. 2012;27(1):58-64.

16. Tsao HM, Wu MH, Higa S, Lee KT, Tai CT, Hsu NW, et al. Anatomic relationship of the esophagus and left atrium: implication for catheter ablation of atrial fibrillation. Chest. 2005;128(4):2581-7.

17. Pappone C, Oral H, Santinelli V, Vicedomini G, Lang CC, Manguso F, et al. Atrio-esophageal fistula as a complication of percutaneous transcatheter ablation of atrial fibrillation. Circulation. 2004;109(22):2724-6.

18. Scanavacca MI, D'Avila A, Parga J, Sosa E. Left atrial-esophageal fistula following radiofrequency catheter ablation of atrial fibrillation. J Cardiovasc Electrophysiol. 2004;15(8):960-2.

19. Wang SL, Ooi CG, Siu CW, Yiu MW, Pang C, Lau CP, et al. Endocardial visualization of esophageal-left atrial anatomic relationship by three-dimensional multidetector computed tomography "navigator imaging". Pacing Clin Electrophysiol. 2006;29(5):502-8.

20. Bai R. Left atrial posterior wall isolation: the icing on the cake. J Interv Card Electrophysiol. 2016;46(3):199-201.

21. Lee JM, Shim J, Park J, Yu HT, Kim TH, Park JK, et al. The Electrical Isolation of the Left Atrial Posterior Wall in Catheter Ablation of Persistent Atrial Fibrillation. JACC Clin Electrophysiol. 2019;5(11):1253-61.

22. Po SS, Nakagawa H, Jackman WM. Localization of left atrial ganglionated plexi in patients with atrial fibrillation. J Cardiovasc Electrophysiol. 2009;20(10):1186-9.

23. Gillinov AM, Pettersson G, Rice TW. Esophageal injury during radiofrequency ablation for atrial fibrillation. J Thorac Cardiovasc Surg. 2001;122(6):1239-40.

24. Sandhu A, Zipse MM, Borne RT, Aleong RG, Tompkins C, Schuller J, et al. Esophageal position, measured luminal temperatures, and risk of atrioesophageal fistula with atrial fibrillation ablation. Pacing Clin Electrophysiol. 2019;42(4):458-63.

25. Piorkowski C, Hindricks G, Schreiber D, Tanner H, Weise W, Koch A, et al. Electroanatomic reconstruction of the left atrium, pulmonary veins, and esophagus compared with the "true anatomy" on multislice computed tomography in patients undergoing catheter ablation of atrial fibrillation. Heart Rhythm. 2006;3(3):317-27. 